\title{
A meta-analysis of psychological interventions for Internet/smartphone addiction among adolescents
}

\author{
ROMUALDAS MALINAUSKAS* and VILIJA MALINAUSKIENE
}

Department of Health, Physical and Social Education, Lithuanian Sports University, Kaunas, Lithuania

(Received: May 21, 2019; revised manuscript received: August 16, 2019; second revised manuscript received: September 26, 2019; accepted: December 2, 2019)

\begin{abstract}
Background and aims: Although the peculiarities of problematic Internet use and Internet addiction have been analyzed previously by researchers, there is still no general agreement in the literature as to the effectiveness of psychological interventions for Internet addiction deployed among adolescents. This study sought to investigate the effects of intervention programs for Internet/smartphone addiction among adolescents through a meta-analysis. Methods: We searched MEDLINE (PubMed), EbscoHost Academic Search Complete, ProQuest, and PsycARTICLES using a combination of "Internet addiction or phone addiction" AND "intervention or treatment" OR "therapy" OR "program" AND "adolescents," and a combination of the following search terms: "patholog_," "problem_," "addict_," “compulsive," “dependen_," “video," “computer," “Internet,” “online," “intervention,” “treat_,” and "therap_." The studies identified during the search were reviewed according to the criteria and a meta-analysis was conducted on the six selected papers published from 2000 to 2019. Only studies with a control/comparison group that performed preintervention and postintervention assessments were included. Results: Included studies showed a trend toward a beneficial effect of intervention on the severity of Internet addictions. The meta-analysis suggested significant effects of all included randomized controlled trials (RCTs) and their educational programs. Conclusions: Psychological interventions may help to reduce addiction severity, but further RCTs are needed to identify the effectiveness of cognitive behavioral therapy. This study provides a basis for developing future programs addressing addiction problems among adolescents.
\end{abstract}

Keywords: adolescents, Internet/smartphone addiction, meta-analysis

\section{INTRODUCTION}

Researchers have investigated the reasons, risks, and severity of Internet/smartphone addiction and interventions for reducing the severity of such in a number of ways and from various disciplinary perspectives. However, even though the peculiarities of problematic Internet use have been previously analyzed by researchers, there is still no general agreement in the literature about the effectiveness of psychological interventions for Internet addiction among adolescents (Chun, Shim, \& Kim, 2017). Therefore, we believe that an assessment of the effectiveness of psychological interventions for Internet addiction among adolescents requires a meta-analytical investigation because Internet addiction is prevalent among adolescents and is associated with various negative outcomes (Mo, Chan, Chan, \& Lau, 2018). Excessive usage of the Internet is associated with a psychiatric condition known as Internet addiction (IA; Pies, 2009; Zhang, Lim, Lee, \& Ho, 2018). Internet use may have negative impacts on daily life function, family relationships, and emotional stability among adolescents (Chun et al., 2017). IA disorder ruins lives by causing neurological complications, psychological disturbances, and social problems (Mo et al., 2018). IA is a complex disorder in terms of its conceptualization, clinical manifestation, and measurement. "Internet Gaming Disorder" was recently included into the fifth edition of Diagnostic and Statistical Manual of Mental Disorders (DSM-5) as a potential new diagnosis (American Psychiatric Association [APA], 2013). The DSM-5 definition of Internet gaming disorder includes nine criteria as follows: (a) preoccupation or obsession with Internet games; (b) appearance of withdrawal symptoms when attempting to cut down or stop Internet gaming; (c) increasing amounts of time must be spent playing the games; (d) unsuccessful efforts to control playing Internet games; (e) loss of interest in other life activities such as hobbies and entertainment; (f) continued overuse of Internet games, despite knowledge of how much Internet gaming is impacting a person's life; (g) lying to others to conceal regarding Internet game use; (h) use of Internet gaming to relieve or escape anxiety or feelings of guilt; and (i) loss of a significant relationship, job, or educational or career

\footnotetext{
* Corresponding author: Dr. Romualdas Malinauskas; Department of Health, Physical and Social Education, Lithuanian Sports University, Sporto 6, 44221 Kaunas, Lithuania; Phone: +370 37 302 672; Fax: +370 37204 515; E-mail: romualdas.malinauskas@ 1su.lt
}

This is an open-access article distributed under the terms of the Creative Commons Attribution-NonCommercial 4.0 International License, which permits unrestricted use, distribution, and reproduction in any medium for non-commercial purposes, provided the original author and source are credited, a link to the CC License is provided, and changes - if any - are indicated. 
opportunity because of Internet gaming (APA, 2013). For the purposes of the present review, IA is operationally defined as a consistent and potentially pathological behavioral pattern characterized by salience (preoccupation with online activities), tolerance (pursuing increasing time to achieve satisfaction), withdrawal symptoms (when unable to use the Internet), using online activities to modify mood, conflict (within oneself, in relationships, or with academic/ occupational activities because of online engagement), and relapse (unsuccessful attempts to control the behavior; Kuss, Shorter, Van Rooij, Van de Mheen, \& Griffiths, 2014).

Considering the available literature (Chun et al., 2017), many studies have identified problems related to IA among adolescents. We employed a description of adolescents in this study based on that by Sawyer, Azzopardi, Wickremarathne, and Patton (2018) in which 10-24 years corresponds more closely to adolescent growth and popular understandings of this life phase and would facilitate extended investments across a broader range of settings. Internet-addicted adolescents are at an increased risk of psychological disorders such as depression (Morgan \& Cotton, 2003), social problems (Shapira, Goldsmith, Keck, Khosla, \& McElroy, 2000), loneliness (Cao \& Su, 2007), and academic issues (Odac1 \& Kalkan, 2010). Internetaddicted adolescents spend less time with their families than do peers without Internet-related addiction (Chun et al., 2017). There are initial findings suggesting also that adolescents suffering from IA might be at a higher risk of suicidal tendencies (Kim et al., 2006).

Given that the severity of IA among adolescents is higher than in other age groups, various new IA programs for adolescents have been developed and implemented (Kim \& Noh, 2019; Yeun \& Han, 2016). Therefore, research focusing on the latest treatments for adolescent IA around the world and which assesses the effectiveness of psychological interventions for reducing the severity of IA among adolescents is required.

\section{Theoretical background}

The results of psychological interventions include desired changes in three areas (Sharma \& Palanichamy, 2018) as follows: (a) symptoms, including physical and mental health symptoms; (b) activities, including but not limited to physical activity, everyday life activities, assigned school and work tasks, maintaining peer relationships, and family rearing and communication activities; and (c) well-being, including spirituality, life satisfaction, quality of life, and the promotion of recovery from IA. Various psychological and behavioral theories have been suggested to explain IA (Sharma \& Palanichamy, 2018).

There is no widely accepted categorization scheme for psychological interventions. The term is generally applied to a broad range of types of interventions, which include psychotherapies [e.g., cognitive behavioral therapy (CBT), interpersonal psychotherapy, and problem-solving therapy], community-based treatment (e.g., assertive community treatment), vocational rehabilitation, peer support services, and integrated care interventions (Sharma \& Palanichamy, 2018). Due to the frequent implementation of CBT, sandplay therapy, and educational programs, there is a need to justify these interventions in particular as appropriate for addressing IA.

The cognitive behavioral model suggests that generalized thinking might contribute to compulsive Internet use in providing a psychological escape mechanism to avoid real or perceived problems (Davis, 2001). This theory of IA is a departure from the other theories in that it emphasizes the individual's cognitions (or thoughts) as the main source of the abnormal behavior. This model posits that IA results from problematic cognitions coupled with behaviors that either intensify or maintain the maladaptive response (Davis, 2001). Research has revealed specific inappropriate thoughts, for example, negative core beliefs that contribute to addictive use of the Internet (Davis, 2001; Sharma \& Palanichamy, 2018). More specifically, the person feels that the Internet is the only place where they feel good about themselves and the world around them (Davis, 2001).

Social skills deficit theory, which is the theoretical background for sandplay therapy and behavior-based educational programming, proposes that individuals' preferences for online rather than face-to-face social interaction play an important role in the development of negative consequences associated with addictive use of the Internet (Caplan, 2003). Adolescents with poor social competence who suffer from psychosocial problems (e.g., depression, social anxiety, and loneliness) or who may be unusually concerned about social interactions are drawn to the anonymity provided by the Internet and the cover it gives for establishing relationships in less dangerous circumstances than those occurring in real life (i.e., face-to-face; Sharma \& Palanichamy, 2018; Shin \& Jang, 2016). Individuals who feel overwhelmed or who experience personal problems or life-changing traumatic events (e.g., divorce, emigration, death, and chronic illness) can develop a preference for online social interactions as an alternative to faceto-face ones and can position themselves in a virtual world.

\section{The present study}

This study sought to investigate the effects of intervention programs for Internet/smartphone addiction among adolescents through a meta-analysis. We assume in this study that different types of treatment programs can have different effects on the severity of IA. Therefore, it is possible through a meta-analysis to empirically determine the strengths of each psychological intervention using the evidence gathered from international literature. Although there are a significant number of studies that discuss the positive impacts of interventions for reducing the severity of IA among adolescents (Chun et al., 2017; Kim \& Noh, 2019), few studies employ a control group and include quantitative data. The meta-analysis conducted in this article provides a quantitative overview of recent developments and findings, enhancing and contributing to the up-to-date literature in this scientific field.

The effectiveness of intervention programs for adolescents is regularly analyzed in systematic reviews and meta-analyses (Kim \& Noh, 2019; Yeun \& Han, 2016). Systematic reviews and meta-analyses distinguish between a variety of interventions that employ various psychological approaches, different frequencies of sessions, and ranging 
lengths in the duration of the intervention process. Taking into consideration the emergence of new randomized controlled trials (RCTs), the aim of this study was to evaluate the effectiveness of interventions used to reduce the IA severity among adolescents, focusing on the appropriateness of the approaches employed to identify significant changes in IA severity.

\section{METHODS}

\section{Data sources}

Relevant articles published in English in academic journals from 2000 to 2019 were collected from four databases: Medline (PubMed), EbscoHost Academic Search Complete, ProQuest, and PsycARTICLES using a combination of "Internet addiction or phone addiction" AND "intervention or treatment" OR "therapy" OR "program" AND "adolescents," and a combination of the following search terms: "patholog_," "problem_," "addict_," "compulsive," "dependen_," "video," "computer," "Internet," "online," "intervention," "treat_," and "therap_." Using this search procedure, a total of 1,464 articles were identified.

After removing duplicates, two independent researchers screened the remaining records based on the titles and abstracts. The full texts of the remaining articles were also reviewed to assess each study's eligibility. Additional reference analysis and searches were conducted manually to avoid overlooking eligible studies. The final included articles were selected by discussion. Data were extracted using a standardized data extraction sheet and included author, published year, study design, total sample size, participants' details, control conditions, intervention characteristics, intervention provider, diagnostic tools of the severity of IA, outcome measures, and study results (Figure 1).

\section{Study selection}

Study selection was based on the use of interventions for IA among adolescents. The present analysis included the following: (a) journal papers and "unpublished" MA theses and $\mathrm{PhD}$ dissertations that provide a quantitative evaluation of the effectiveness of intervention( $s$ ) for reducing the severity of IA; (b) studies targeting adolescents aged 10-24 years; (c) studies with publication dates between 2000 and 2019, since IA intervention approaches are a relatively nascent field within the literature; and (d) fulltext studies published only in the English language. In contrast, the following were excluded: (a) studies referring to IA but not covering intervention(s) for IA; (b) studies with the main text not published in the English language (e.g., only tables were in English); (c) studies that do not provide indicators of the severity of IA; and (d) cyberbullying and gambling prevention studies, as these do not have IA or gaming as their primary intervention foci.

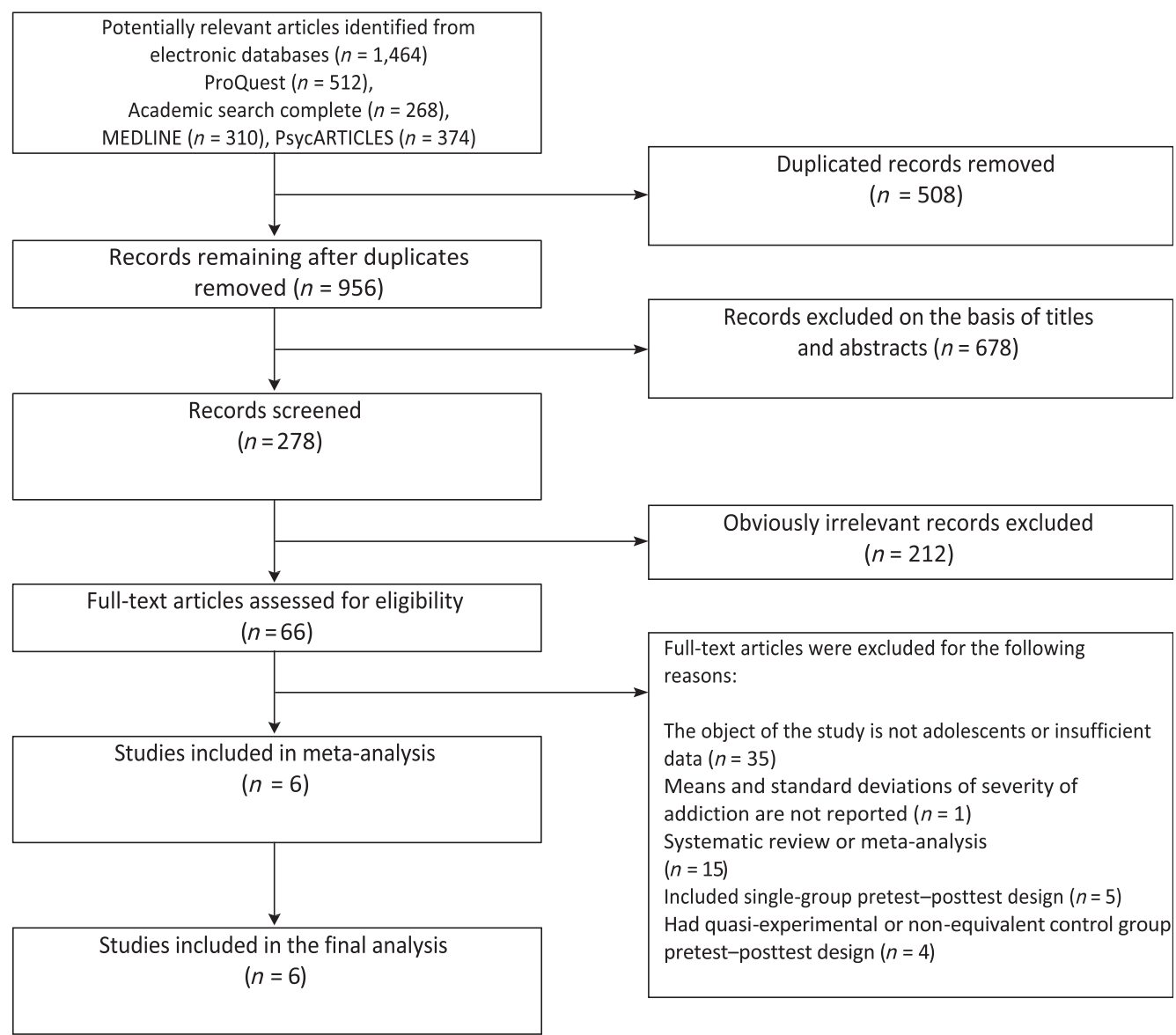

Figure 1. A flowchart of study inclusion and exclusion 


\section{Study inclusion}

To be eligible for the meta-analysis, studies had to meet the following inclusion criteria: (a) an RCT study design, (b) participants met the diagnostic criteria for IA, (c) no interventions or usual care were given to the control group, (d) the study objective was to evaluate the effects of treatment on IA, and (e) the study included psychological or comprehensive programs that implemented several different types of treatments intended to reduce IA severity.

Studies were excluded if they fulfilled any of the following criteria: (a) the study was a single-group pre-post comparison study, (b) the study used a quasi-experimental or non-equivalent control group pretest-posttest design, (c) the full text of the study was unavailable, or (d) the study did not provide sufficient data (i.e., mean and standard deviations of the severity of addiction were not reported). Based on these criteria, studies were selected for inclusion in the meta-analysis.

\section{Data extraction and quality assessment}

To determine the effect sizes of the treatment interventions reported, the sample sizes of the experimental and control groups as well as the averages and standard deviations of the severity of IA-related pre- and posttest outcomes were coded as dependent variables. The theoretical interventional models included CBT, sandplay therapy, or behavior-based educational programming. Studies were also analyzed for the type of control (e.g., waiting list, no treatment, and treatment as usual). The relevant data were extracted by the two authors. The authors (raters) then coded these data independently. Disagreements between raters were resolved by consensus. The interrater agreement between the two raters regarding the included studies was satisfactory $(>81 \%)$.

The methodological quality of information was assessed in this study with a scale based upon criteria developed by the APA for assessing empirically validated interventions (Gingerich \& Eisengart, 2000; Jackson, Hodge, \& Vaughn, 2010). The six standards used to assess methodological rigor were (a) randomization of sample; (b) comparison with other treatments, standard services, or waitlist control; (c) definition of population; (d) use of validated and reliable outcome measures; (e) use of treatment manuals or curriculum; and (f) large sample size (i.e., more than 25 study subjects per group). One point was given for the presence of each standard. Thus, each study received a score of $0-6$ points, with higher scores indicating the existence of higher levels of methodological quality.

All six studies were independently rated by two authors. To assess interrater reliability, Cohen's (1960) $\kappa$ coefficient was used. In this approach, a value of 0 indicates a level of agreement that would be expected based upon chance alone, whereas a value of 1 indicates perfect agreement. The $\kappa$ coefficient obtained in this study was 1. As such, the strength of agreement can be interpreted as very good according to Altman (1991). The quality scores are presented in Table 1.

\section{Outcomes measurement}

Tools used to assess the severity of IA varied between studies and employed various questionnaire scales such as the Internet Overuse Self-rating Scale, the Young Internet Addiction Scale, the Problematic Internet Use Scale, the Youth Smartphone Addiction Self-report Scale, the Internet Addiction Scale, and the Bergen Social Media Addiction Scale. Psychological indicators were assessed with Beard's Diagnostic Questionnaire, the Screen for Child Anxietyrelated Emotional Disorders, the Strength and Difficulties Questionnaire, the Time Management Disposition Scale, the Beck Anxiety Inventory, the Beck Depression Inventory, the Life Satisfaction Scale, the School Problematic Behaviour Scale, the Self-esteem Scale, the Adapted General Health Questionnaire, the Utrecht Work Engagement Scale Student, and the evaluation of both online game-playing time (hours/week) and sleep quality.

\section{Data analysis}

For the selected studies that utilized a control group design, the intervention group included all participants who received psychological treatment and the control group consisted of all participants who did not. Outcome means and standard deviations were input from the available descriptive data. Severity changes were used to assess the effect size of the effectiveness of the treatment(s) assessed. For each target population and defined indicator of the severity of IA, pooled estimates and $95 \%$ confidence intervals (CI) of effect sizes were calculated using an inverse variance-weighted, random-effects meta-analysis model (Hedges \& Olkin, 1985). Between-study heterogeneity was quantified with the $I^{2}$ statistic (Higgins \& Thompson, 2002), which indicates the true effect size variance as a proportion of the total variance in a study estimate due to heterogeneity. To test for heterogeneity, we also calculated Cochran's $Q$ statistic, which considers the degrees of freedom. For the null hypothesis (which posits that all effect sizes are equal) to be rejected, Cochran's $Q$ statistic must be statistically significant, while the proportion of the error variance among the total variance observed from the effect sizes must be significantly higher than expected, given sampling errors (Borenstein, Hedges, Higgins, \& Rothstein, 2009). The degree of heterogeneity was assessed using the formula: $I^{2}=100 \% \times[Q-(k-1)] / Q$, where $k$ represents the number of studies included. The $I^{2}$ statistic ranges from $0 \%$ to $100 \%$, and cut-off values of $25 \%, 50 \%$, and $75 \%$ are used to indicate low, moderate, and high degrees of heterogeneity, respectively.

Included studies were also grouped according to intervention type to assess the effectiveness of the interventions. In addition, subgroup meta-analyses were conducted. All meta-analyses were performed using MedCalc Statistical Software (ISI ResearchSoft, Berkeley, CA, USA).

\section{Ethics}

The study procedures were carried out in accordance with the Declaration of Helsinki. Ethical clearance was obtained 
Interventions for Internet addiction: A meta-analysis

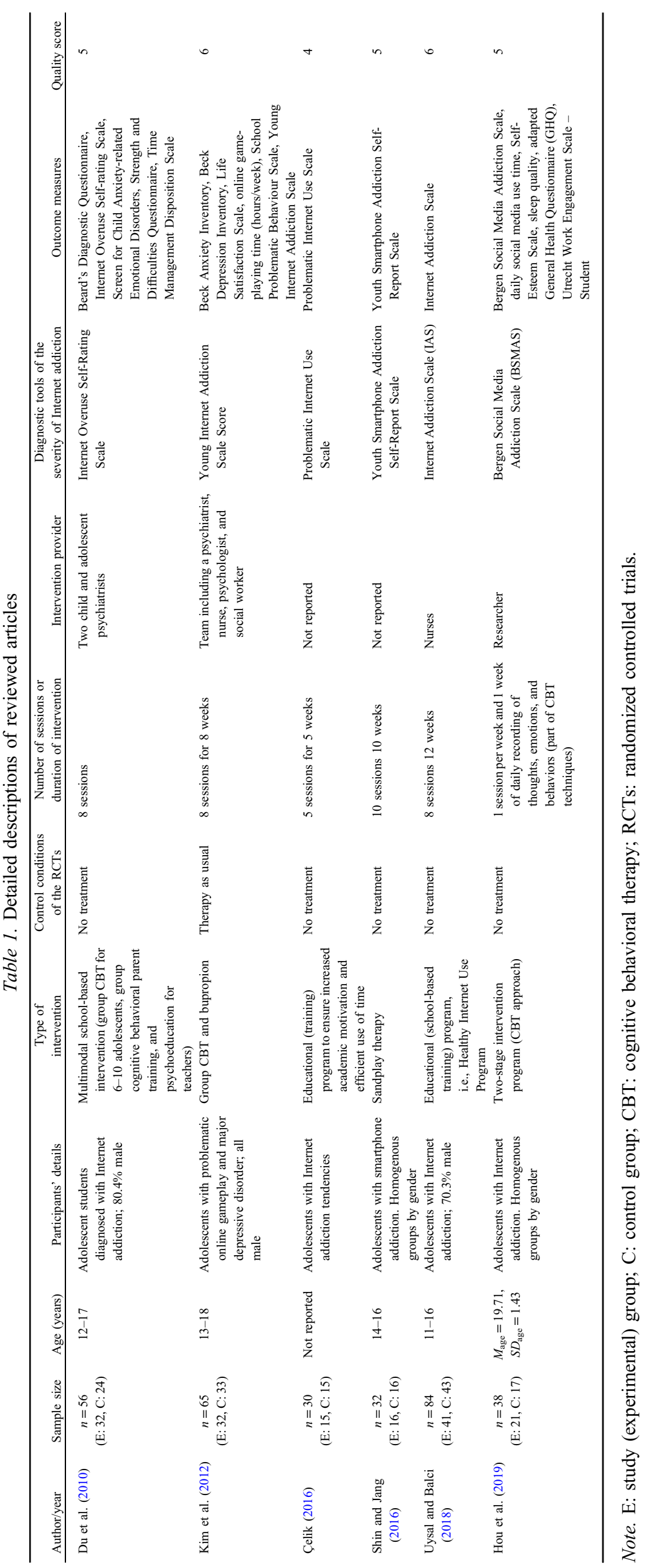


from the Committee for Social Sciences Research Ethics of the Lithuanian Sports University.

\section{RESULTS}

\section{Study characteristics}

Six publications met the meta-analysis criteria, in which all studied the effects of intervention programs for IA among adolescents. Five studies were conducted by researchers at Asian universities (Çelik, 2016; Du, Jiang, \& Vance, 2010; Hou, Xiong, Jiang, Song, \& Wang, 2019; Kim, Han, Lee, \& Renshaw, 2012; Shin \& Jang, 2016) and one study was performed at a European university (Uysal \& Balci, 2018). Meta-analyses were performed separately on RCTs, CBT studies, and educational programs (Table 1). All studies included in the meta-analysis were RCTs. Studies with a non-equivalent control group pretest-posttest design and studies with a single-group pretest-posttest design were not included in the meta-analysis. Observed interventions of the included studies were CBT, sandplay therapy, and behaviorbased educational programming. In particular, three studies incorporated CBT (Du et al., 2010; Hou et al., 2019; Kim et al., 2012), one study incorporated sandplay therapy (Shin \& Jang, 2016), and two studies incorporated educational programming (Çelik, 2016; Uysal \& Balci, 2018).

Four studies targeted participants with general IA (Çelik, 2016; Du et al., 2010; Hou et al., 2019; Uysal \& Balci, 2018), one study targeted participants with Internet gaming addiction (Kim et al., 2012), and one targeted adolescents with smartphone addiction (Shin \& Jang, 2016). A total of 305 adolescents aged 12-21 years old participated in the studies. One of the three CBT studies reported only the mean age of participants (Hou et al., 2019) and one did not report on an adolescent age range (Çelik, 2016). Most of the included studies involved predominantly male participants; in further detail, the participants in all studies were mostly males, with only one study (Hou et al., 2019) featuring an equal number of male and female participants. All included studies measured the severity of IA but did not mention cut-off points of the scales that they used; therefore, it was impossible to accurately classify the severity of addiction (i.e., mild, moderate, or severe).

\section{Intervention characteristics}

The characteristics of interventions are presented in Table 1. There was substantial variability in the number of sessions, ranging from 1 (Hou et al., 2019) to 10 (Shin \& Jang, 2016). The mean total number of sessions across all included psychological interventions was 6.7 sessions.

Four studies mentioned intervention providers, which were categorized as psychiatrists (Du et al., 2010); researchers (Hou et al., 2019); nurses (Uysal \& Balci, 2018); or a multidisciplinary team including a psychiatrist, nurse, psychologist, and social worker (Kim et al., 2012). Regarding nurses' roles, one study that conducted CBT mentioned that a nurse conducted two sessions (out of eight; Kim et al., 2012). The face-to-face intervention format was used in all studies included in this study. All six studies also used group interventions; however, only one of the included studies that used a group format stated the number of groups included (Du et al., 2010). The study of a multimodal school-based intervention used four intervention groups with 6-10 individuals included in each group for a total of 32 individuals included in the intervention condition (Du et al., 2010).

In one randomized trial (Du et al., 2010), participants in the treatment group were treated with an eight-session multimodal school-based group CBT approach, whereas participants in the control group received no intervention. Internet Overuse Self-Rating Scale scores did not differ between the active and control groups at baseline, immediately after the intervention, or at 6 months later. Both groups showed a decrease in Internet use immediately after the intervention and at 6 months after when compared before the intervention (Du et al., 2010). The active group's effect sizes both immediately and at 6 months of follow-up, however, were large (Cohen's $d=1.08$ and 1.35) when compared with those of the control group (Cohen's $d=0.66$ and 0.67; Du et al., 2010). Furthermore, although Internet use decreased in both groups, only the multimodal schoolbased CBT group showed improved time management skills and better emotional, cognitive, and behavioral symptoms (Du et al., 2010).

A second randomized trial compared eight sessions of CBT plus bupropion to bupropion alone for adolescents diagnosed with IA (Kim et al., 2012). There were no statistically significant between-group differences in reductions in depression symptoms. The CBT group showed significantly greater reductions in time spent gaming and IA symptoms as compared with the medication-only group in the posttreatment period, and this effect was maintained at 4 weeks posttreatment. However, there was no control for therapist time implemented (i.e., CBT sessions lasted between 90 and $120 \mathrm{~min}$ weekly, whereas medication management consisted of 10-min weekly check-ins).

In the most recent randomized trial, participants in the treatment group were treated with a two-stage intervention program (CBT approach) and participants in the control group received no intervention. The first stage involved cognitive reconstruction and took approximately $30 \mathrm{~min}$ (Young, 1999). During the second stage of the intervention (duration of 1 week), participants in the experimental group were asked to keep a daily log to record their thoughts, emotions, and behaviors related to social media use as part of the CBT program. This intervention program with a CBT-based approach effectively reduced social media addiction and improved students' mental health and learning efficiency (Hou et al., 2019).

One study that incorporated sandplay therapy (Shin \& Jang, 2016) observed that group sandplay therapy was effective at helping with IA. A comparison of the addiction level before and after group sandplay therapy showed a statistical significance; specifically, the study demonstrated that group sandplay therapy is effective in reducing adolescents' Internet dependence.

Two of the included studies used educational programming (Çelik, 2016; Uysal \& Balci, 2018). The general aims of the first educational program (Çelik, 2016) were to reduce the study group members' time spent on the Internet, to improve their awareness by increasing their academic 
motivation levels and promoting a more efficient use of their time, and to mitigate IA tendencies through group experiences. Ultimately, the results revealed that the educational program was effective at reducing adolescent's IA tendencies (Çelik, 2016). Separately, the general aims of the second educational program (Healthy Internet Use Program) were to develop new healthy Internet use concepts and habits, to improve existing healthy Internet use habits and promote healthy lifestyle choices, and to learn to organize daily activities better (Uysal \& Balci, 2018). The findings here suggested that the implementation of the Healthy Internet Use Program decreased the rate of IA among adolescents.

\section{Effects of psychological interventions on addiction severity}

Six RCTs reported mean and standard deviations results of the severity of IA in both experimental and control groups in the posttest period. Statistical indicators (means and standard deviations) of the severity of IA among adolescents in the included studies are given in Table 2.

A meta-analysis of the six RCTs showed that experimental participants who were provided with psychological interventions had a significantly lower $(p=.00)$ severity of IA than did control group at posttest [standardized mean difference $(\mathrm{SMD})=-0.67,95 \% \mathrm{CI}=-1.07$ to -0.27 ; Table 3; Figure 2). For the RCT group, statistically significant heterogeneity was observed $\left(I^{2}=64.63 \% ; \quad p=.01\right.$; $Q=14.14$ ), so a random-effects model was used.

\section{Effects of CBT on addiction severity}

A meta-analysis of the three RCTs for which there were sufficient data (Du et al., 2010; Hou et al., 2019; Kim et al., 2012) showed no significant variations between the CBT and control groups with regard to the severity of IA (SMD: $-0.60,95 \%$ CI: -1.38 to 0.19 ; Table 3 ; Figure 3 ). The results revealed that a high degree of heterogeneity was present in the severity of addiction estimates $\left(I^{2}=82.53 \%\right.$; $p=.00 ; Q=11.45)$ and the random-effects model was used. The analysis on group CBT showed that there was a reduction in the severity of IA, with a SMD of -0.60 points, but this was not statistically significant $(p=.14)$. The $95 \%$ CI for the SMD was -1.38 to 0.19 .

\section{Effects of educational programming on addiction severity}

A meta-analysis of the two RCTs that included educational programming groups showed that experimental participants who were provided with this type of intervention had a significantly lower $(p=.02)$ severity of IA as compared with those in the control group at posttest (SMD: $-0.76,95 \% \mathrm{CI}$ for the SMD: -1.38 to -0.13 ; Table 3; Figure 3). For the educational programming studies group, an insignificant degree of heterogeneity was observed $\left(I^{2}=52.72 \% ; p=.15\right.$; $Q=2.12$ ), so a random-effects model was deployed. With respect to the quantification of heterogeneity in a metaanalysis (Higgins \& Thompson, 2002), the heterogeneity of effects across studies is considered notable when $I^{2}$ exceeds

Table 2. Severity of addiction among adolescents in studies included in the meta-analyses of all included RCTs, RCTs with CBT, and RCTs with educational programming

\begin{tabular}{|c|c|c|c|c|c|c|c|}
\hline \multirow[b]{2}{*}{ Meta-analysis ID } & \multirow[b]{2}{*}{ Author/year } & \multicolumn{3}{|c|}{ SG } & \multicolumn{3}{|c|}{ CG } \\
\hline & & $N$ & Mean & $S D$ & $N$ & Mean & $S D$ \\
\hline \multicolumn{8}{|l|}{ All RCTs } \\
\hline 1 & Du et al. (2010) & 32 & 23.14 & 11.88 & 24 & 21.40 & 13.26 \\
\hline 2 & Kim et al. (2012) & 15 & 33.60 & 11.10 & 33 & 43.50 & 16.20 \\
\hline 3 & Çelik (2016) & 16 & 89.06 & 17.49 & 15 & 107.25 & 12.80 \\
\hline 4 & Shin and Jang (2016) & 41 & 39.25 & 9.94 & 16 & 45.12 & 4.10 \\
\hline 5 & Uysal and Balci (2018) & 21 & 76.41 & 13.85 & 43 & 84.91 & 18.72 \\
\hline 6 & Hou et al. (2019) & 32 & 14.62 & 3.72 & 17 & 19.18 & 3.07 \\
\hline \multicolumn{8}{|l|}{ RCTs with $C B T$} \\
\hline 1 & Du et al. (2010) & 32 & 23.14 & 11.88 & 24 & 21.40 & 13.26 \\
\hline 2 & Kim et al. (2012) & 32 & 33.60 & 11.10 & 33 & 43.50 & 16.20 \\
\hline 6 & Hou et al. (2019) & 21 & 14.62 & 3.72 & 17 & 19.18 & 3.07 \\
\hline \multicolumn{8}{|c|}{ RCTs with educational programming } \\
\hline 3 & Çelik (2016) & 16 & 89.06 & 17.49 & 15 & 107.25 & 12.80 \\
\hline 5 & Uysal and Balci (2018) & 21 & 76.41 & 13.85 & 43 & 84.91 & 18.72 \\
\hline
\end{tabular}

Note. SD: standard deviation; SG: study group; CG: control group; RCTs: randomized controlled trials; CBT: cognitive behavioral therapy; EP: educational program.

Table 3. Results of meta-analyses

\begin{tabular}{lcccccc}
\hline Group & Studies & $Q$ value & Heterogeneity $p$ & $I^{2}(\%)$ & $p$ of meta-analysis & SMD [95\% CI] Random effects \\
\hline RCTs & 6 & 14.14 & .01 & 64.63 & .00 & $-0.67[-1.07,-0.27]$ \\
RCTs with CBT & 3 & 11.45 & .00 & 82.53 & .14 & $-0.60[-1.38,0.19]$ \\
RCTs with EP & 2 & 2.12 & .15 & 52.72 & .02 & $-0.76[-1.38,-0.13]$ \\
\hline
\end{tabular}

Note. SG: study group; CG: control group; RCTs: randomized controlled trials; CBT: cognitive behavioral therapy; EP: educational program; CI: confidence interval; SMD: standardized mean difference. 


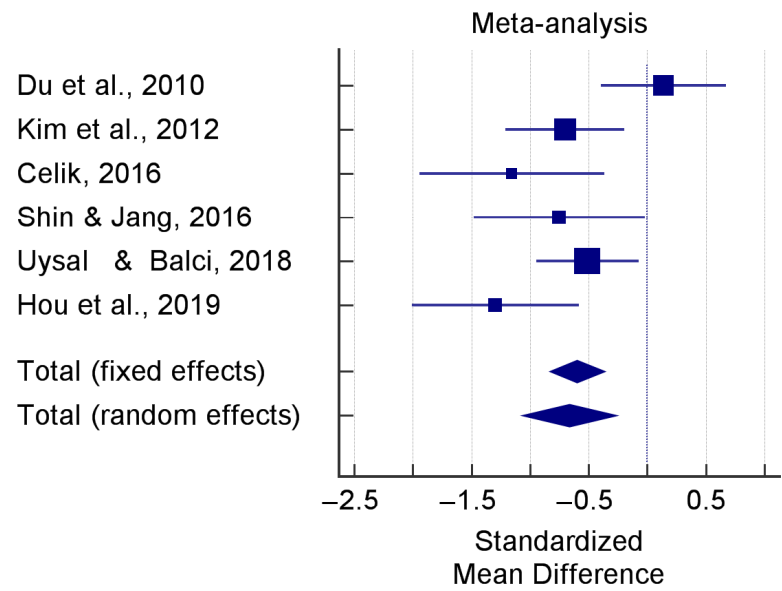

Figure 2. Effects of psychological interventions of included RCTs for reducing the severity of Internet addiction

$60 \%$. The interventions with a large effect size on the reduction of severity of IA were educational (training) programming (SMD: $-1.16,95 \% \mathrm{CI}:-1.94$ to -0.37 ) and educational (school-based training) programming (SMD: $-0.51,95 \%$ CI: -0.95 to -0.07 ; Figures 3 and 4 ).

For the reduction of the severity of IA, educational (training) programming to ensure increased academic motivation and more efficient time usage (Çelik, 2016) had a larger effect than did educational (school-based training) programming implemented to ensure healthy Internet use (Uysal \& Balci, 2018).

Funnel plots were developed (Figure 5). Egger's regression test and Egger's and Begg-Mazumdar rank-correlation tests for funnel plot asymmetry were performed to assess the publication bias in the meta-analysis. No significant publication bias was found for any of the included RCTs [Egger's regression test for funnel plot asymmetry: $t(4)=-1.41$; $p=.231]$. Separately, the Begg-Mazumdar rank-correlation test for funnel plot asymmetry (Kendall's $\tau=-0.33$; $p=.469$ ) indicated symmetry (Figure 5A). However, two open circles on the right side show missing NULL studies estimated with Duval and Tweedie's trim-and-fill method, added in the funnel plot (Figure 5A). Fail-safe $N$ calculation

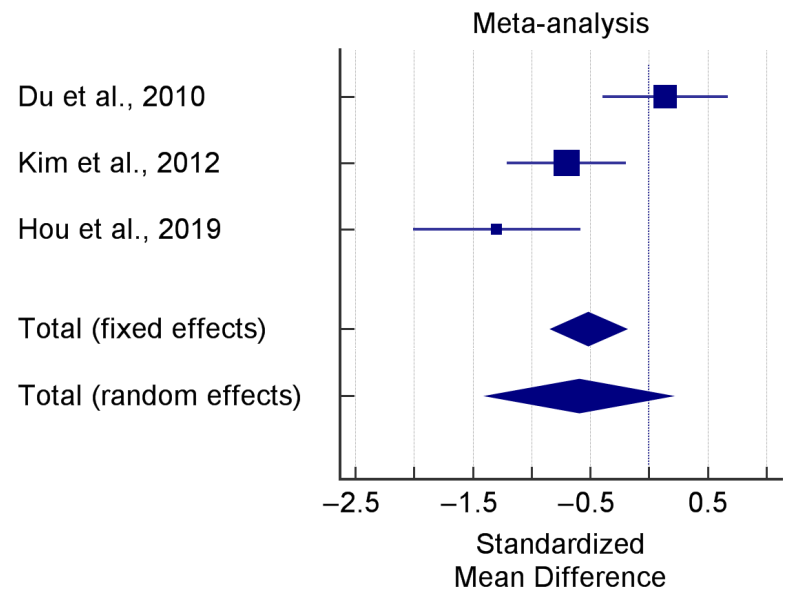

Figure 3. Effects of psychological interventions of RCTs with CBT for reducing the severity of Internet addiction

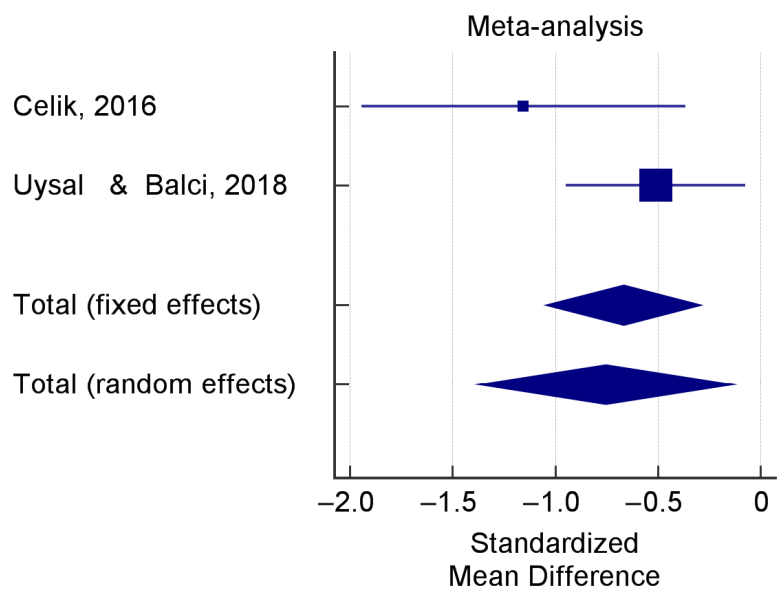

Figure 4. Effects of psychological interventions of RCTs with educational programming for reducing the severity of Internet addiction

using the Rosenthal approach showed that the fail-safe $N$ is 58. For clarification, the fail-safe $N$ is the number of non-significant studies necessary to make the result nonsignificant, and this number is robust when $N>5 n+10$ (Rosenberg, 2005). "When the fail-safe $N$ is high, [such] is interpreted to mean that even a large number of nonsignifcant studies may not influence the statistical significance of meta-analytic results too greatly" (Oswald \& Plonsky, 2010).

As shown in Figure 5B, the nearly symmetrical funnel plot for the RCTs including CBT indicates an absence of publication bias. The results from the Egger's regression test $[t(1)=-0.78 ; p=.578]$ and from the Begg-Mazumdar rank-correlation test for funnel plot asymmetry (Kendall's $\tau=-0.33 ; p=1.00$ ) confirmed that there was no significant asymmetry with respect to the funnel plot. These data indicate that there was no significant publication bias in the meta-analysis performed involving RCTs containing CBT as an intervention. The fail-safe number $N$ calculated here using the Rosenthal approach was not robust $(N=10)$.

Separately, a funnel plot was made (Figure 5C) and Egger's and Begg-Mazumdar tests were performed to assess publication bias in the meta-analysis of RCTs containing educational programming. Ultimately, both the results from the Egger's regression test (Egger's test, not applicable) and those from the Begg-Mazumdar rankcorrelation test for funnel plot asymmetry (Kendall's $\tau=1.00 ; p=1.00)$ suggested that publication bias was present in this particular meta-analysis. Thus, we used the aforementioned trim-and-fill method to correct the funnel asymmetry caused by publication bias. One open circle on the right side shows a missing NULL study estimated using the trim-and-fill method, added in the funnel plot (Figure 5C). The fail-safe number $N$ calculated using the Rosenthal approach here was not robust $(N=9)$.

\section{DISCUSSION}

With the present meta-analysis, we gathered scientific evidence about how psychological interventions for reducing 

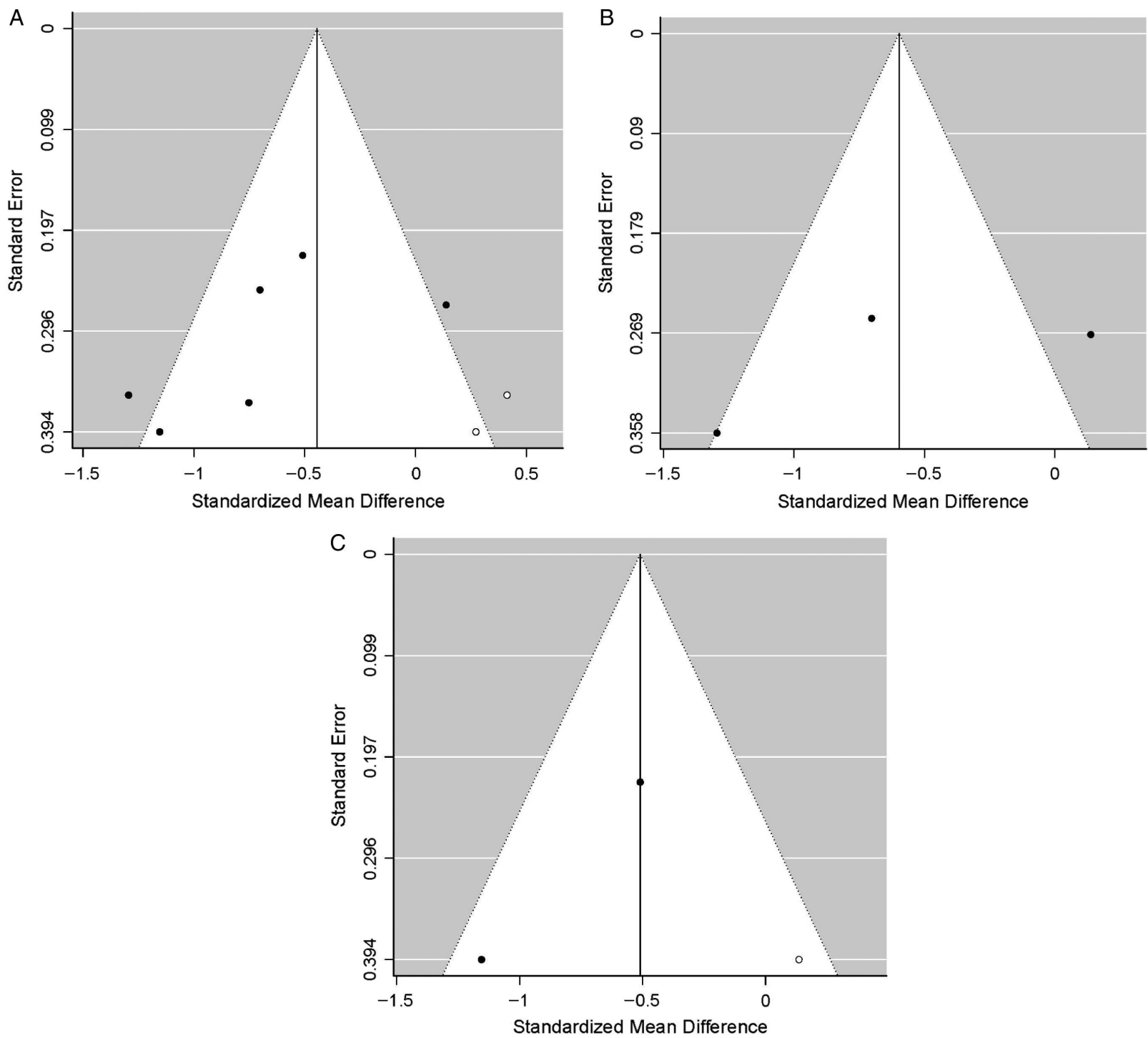

Figure 5. Funnel plots of the included studies. (A) Funnel plot of all included RCTs. (B) Funnel plot of RCTs with CBT. (C) Funnel plot of RCTs with educational programming

the severity of IA are effective. The analysis of the effectiveness of the included interventions for reducing the severity of IA among adolescents showed a trend toward beneficial effects of the deployed intervention(s) on the severity of IAs. The interventions conducted in the reviewed articles included CBT, sandplay therapy, and educational programming. All studies included in this analysis were developed for a group. Evidence from the present study is in agreement with the results of other studies (Du, Jiang, \& Vance, 2010; Park, 2009; Yeun \& Han, 2016) and suggests that group intervention appears to be the predominant and effective modality for treating IA.

A meta-analysis of the six included RCTs showed that experimental participants who were provided with psychological interventions had a significantly lower severity of IA than the control group at posttest, although statistically significant heterogeneity was observed among the studies. The results of a meta-analysis of the two studies with educational programming interventions (Çelik, 2016; Uysal \& Balci, 2018) showed that educational programs (e.g., educational training program, educational school-based training program) have statistically significant effects on reducing the severity of IA. The analysis of a group CBT approach showed that there was similarly a reduction in the severity of IA with this method, but not in a statistically significant manner. In summary, the findings of this study suggest that different types of interventions might reduce the severity of IA among adolescents to varying degrees.

Of note, this is not the first meta-analysis to individually investigate the association between psychological interventions and the severity of IA with consideration of heterogeneity (Kim \& Noh, 2019). In the study by Kim and Noh (2019), the results were similar: a meta-analysis of the four RCTs showed that experimental participants who were provided with psychological interventions had a significantly lower severity of addiction as compared with in the control group at posttest. However, evidence supporting which intervention is most effective in reducing the severity of addiction was limited (Kim \& Noh, 2019).

CBT has been identified to be effective in treating IA in many studies (Du, Jiang, \& Vance, 2010; Yeun \& Han, 2016). However, although CBT was used the most in the 
assembled articles in this study, we cannot conclude that CBT may be more effective than the other psychological interventions considered. For instance, the results of the meta-analysis by Kim and Noh (2019) of the two studies that employed group CBT (Du et al., 2010; Kim et al., 2012) did not support the presence of a significant effect on the severity of addiction. Furthermore, the results of the present meta-analysis of the three studies that employed group CBT (Du et al., 2010; Hou et al., 2019; Kim et al., 2012) also showed that there was no significant difference between the CBT and control groups with regard to the severity of IA, so we can conclude that the results of the present meta-analysis do not support that group CBT had significant effects on the severity of IA.

In the present meta-analysis, the overall effect size was found to be moderate for all six RCTs $(\mathrm{SMD}=-0.67)$ and, for the educational programming group, in reducing the severity of addiction ( $\mathrm{SMD}=-0.76$ ). The overall obtained results of the effect size of psychological interventions should be interpreted critically, as data from only six studies were included in this meta-analysis. However, one strength is that all six studies in this meta-analysis were rated as being of high quality. Earlier meta-analyses and systematic reviews in which the impacts of psychological interventions on the severity of IA among adolescents were analyzed were usually only of acceptable quality in that they usually included only small population samples, small numbers of sessions, or short durations of interventions (Davey \& Davey, 2014; Koo \& Kwon, 2014) and, currently, most attention is paid to studies that meet the highest standards of quality (Liu, Nie, \& Wang, 2017; Vondráčková \& Gabrhelik, 2016).

Regarding the effects of intervention according to duration, two-thirds of those studies included had eight or more intervention sessions. This group thus exhibited statistically significant effects for the severity of IA (SMD $=-0.44$, $95 \% \mathrm{CI}$ for the $\mathrm{SMD}=-0.84$ to -0.05 ). A previous review of psychosocial interventions analyzing such outcomes among children demonstrated that longer interventions yielded statistically significant effects (Yeun \& Han, 2016). Similarly, this meta-analysis verified the efficacy of psychological interventions for reducing the severity of IA by conducting more than seven sessions, which included moderate effect sizes, among adolescents. Therefore, because a certain time period is necessary to change the recognition and behaviors of participants, long-term programs for reducing the severity of IA might be more effective than short-term programs (Oh \& Kim, 2009). The previous metaanalysis by Liu et al. (2017) also identified the robustness of the efficacy of long-term programs for reducing the severity of IA.

The results of the present meta-analysis are similar to those of other meta-analyses (Liu et al., 2017) that evaluated the efficacy of psychological interventions for reducing the severity of IA. The results of the meta-analysis by Liu et al. (2017), in which all included studies were conducted in adolescents, showed that the overall effect of CBT on the severity of IA is of a large size ( $\mathrm{SMD}=-1.88,95 \%$ $\mathrm{CI}=-2.53$ to $-1.23 ; p<.01)$. Similarly, a meta-analysis of four RCTs (Kim \& Noh, 2019) showed that experimental participants who were provided with psychological interventions had a significantly lower severity of IA than those in the control group at posttest, demonstrating that the overall effect of the RCTs on the severity of IA is large $(\mathrm{SMD}=-1.47,95 \% \mathrm{CI}=-2.71$ to $-0.23 ; p<0.01)$.

\section{Limitations and future research}

The main strength of this research is that all studies included in the meta-analysis were RCTs. The second advantage of this meta-analysis is that it provided a comprehensive analysis of the effectiveness of interventions with respect to the indicators used to measure the severity of IA. However, the present meta-analysis also has some limitations. First, only publications published in the English language were included. Second, of the six included studies, five were conducted in Asian countries and one was conducted in Europe; therefore, further intervention studies in other countries (e.g., in North and South America) are needed to better generalize our results globally. Third, there was substantial variability in the number of intervention sessions, ranging from 1 to 10 , and future researchers may want to consider including more than one session. Fourth, in two of the included six studies, intervention providers were not reported. Therefore, further research should consider intervention providers' characteristics and their influence on the treatment of IA. Fifth, for the RCTs with CBT group, statistically significant heterogeneity was observed, which was partially explained by differences in the number of sessions and by differences in the diagnostic tools of the severity of IA used. More RCTs for reducing the severity of IA should be conducted to combine data quantitatively without statistically significant heterogeneity. Future research should also focus on people who are part of the formative environment of adolescents who are at risk of IA such as parents, teachers, peers, and others close to them.

\section{CONCLUSIONS}

The present meta-analysis showed significant effects of all included RCTs and RCTs that contained educational programming as an intervention. Psychological interventions may help to reduce addiction severity, but further RCTs are needed to identify the effectiveness of CBT. This study provides a basis for developing future programs addressing IA problems among adolescents.

Funding sources: No financial support was received for this study.

Authors' contribution: RM contributed to study design, statistical analysis, data interpretation, manuscript preparation, and literature search. VM involved in statistical analysis, manuscript preparation, and literature search.

Conflict of interest: The authors declare no conflict of interest. 


\section{REFERENCES}

Altman, D. G. (1991). Practical statistics for medical research. London, UK: Chapman and Hall.

American Psychiatric Association [APA]. (2013). Diagnostic and statistical manual of mental disorders (5th ed.). Washington, DC: American Psychiatric Association.

Borenstein, M., Hedges, L. V., Higgins, J. P., \& Rothstein, H. R. (2009). Introduction to meta-analysis. WestSussex, UK: John Wiley \& Sons Ltd.

Cao, F., \& Su, L. (2007). Internet addiction among Chinese adolescents: Prevalence and psychological features. Child: Care, Health and Development, 33(3), 275-281. doi:10.1111/ j.1365-2214.2006.00715.x

Caplan, S. E. (2003). Preference for online social interaction: A theory of problematic Internet use and psychosocial wellbeing. Communication Research, 30(6), 625-648. doi:10. $1177 / 0093650203257842$

Çelik, Ç. B. (2016). Educational intervention for reducing Internet addiction tendencies. The Turkish Journal on Addiction, 3(3), 175-186. doi:10.15805/addicta.2016.3.0021

Chun, J., Shim, H., \& Kim, S. (2017). A meta-analysis of treatment interventions for Internet addiction among Korean adolescents. Cyberpsychology, Behavior, and Social Networking, 20(4), 225-231. doi:10.1089/cyber.2016.0188

Cohen, J. (1960). A coefficient of agreement for nominal scales. Educational and Psychological Measurement, 20(1), 37-46. doi: $10.1177 / 001316446002000104$

Davey, S., \& Davey, A. (2014). Assessment of smartphone addiction in Indian adolescents: A mixed method study by systematic-review and meta-analysis approach. International Journal of Preventive Medicine, 5(12), 1500-1511. doi:10. 11648/j.ijnfs.20150404.12

Davis, R. A. (2001). A cognitive-behavioral model of pathological Internet use. Computers in Human Behavior, 17(2), 187-195. doi:10.1016/S0747-5632(00)00041-8

Du, Y. S., Jiang, W., \& Vance, A. (2010). Longer term effect of randomized, controlled group cognitive behavioural therapy for Internet addiction in adolescent students in Shanghai. Australian and New Zealand Journal of Psychiatry, 44(2), 129-134. doi:10.3109/00048670903282725

Gingerich, W. J., \& Eisengart, S. (2000). Solution-focused brief therapy: A review of the outcome research. Family Process, 39(4), 477-498. doi:10.1111/j.1545-5300.2000.39408.x

Hedges, L., \& Olkin, I. (1985). Statistical models for metaanalysis. New York, NY: Academic Press.

Higgins, J. P., \& Thompson, S. G. (2002). Quantifying heterogeneity in a meta-analysis. Statistics in Medicine, 21(11), 15391558. doi:10.1002/sim. 1186

Hou, Y., Xiong, D., Jiang, T., Song, L., \& Wang, Q. (2019). Social media addiction: Its impact, mediation, and intervention. Cyberpsychology: Journal of Psychosocial Research on Cyberspace, 13(1), 4. doi:10.5817/CP2019-1-4

Jackson, K. F., Hodge, D. R., \& Vaughn, M. G. (2010). A metaanalysis of culturally sensitive interventions designed to reduce high-risk behaviors among African American youth. Journal of Social Service Research, 36(3), 163-173. doi:10.1080/ 01488371003697780

Kim, S. M., Han, D. H., Lee, Y. S., \& Renshaw, P. F. (2012). Combined cognitive behavioral therapy and bupropion for the treatment of problematic on-line game play in adolescents with major depressive disorder. Computers in Human Behavior, 28(5), 1954-1959. doi:10.1016/j.chb.2012.05.015

Kim, S., \& Noh, D. (2019). The current status of psychological intervention research for Internet addiction and Internet gaming disorder. Issues in Mental Health Nursing, 1, 1-7. doi:10.1080/ 01612840.2018.1534910

Kim, K., Ryu, E., Chon, M. Y., Yeun, E. J., Choi, S. Y., Seo, J. S., \& Nam, B. W. (2006). Internet addiction in Korean adolescents and its relation to depression and suicidal ideation: A questionnaire survey. International Journal of Nursing Studies, 43(2), 185-192. doi:10.1016/j.ijnurstu. 2005.02.005

Koo, H. J., \& Kwon, J. H. (2014). Risk and protective factors of Internet addiction: A meta-analysis of empirical studies in Korea. Yonsei Medical Journal, 55(6), 1691-1711. doi:10. 3349/ymj.2014.55.6.1691

Kuss, D. J., Shorter, G. W., Van Rooij, A. J., Van de Mheen, D., \& Griffiths, M. D. (2014). The Internet addiction components model and personality: Establishing construct validity via a nomological network. Computers in Human Behavior, 39, 312-321. doi:10.1016/j.chb.2014.07.031

Liu, J., Nie, J., \& Wang, Y. (2017). Effects of group counseling programs, cognitive behavioral therapy, and sports intervention on Internet addiction in East Asia: A systematic review and meta-analysis. International Journal of Environmental Research and Public Health, 14(12), 1470. doi:10.3390/ ijerph14121470

Mo, P. K., Chan, V. W., Chan, S. W., \& Lau, J. T. (2018). The role of social support on emotion dysregulation and Internet addiction among Chinese adolescents: A structural equation model. Addictive Behaviors, 82, 86-93. doi:10.1016/j.addbeh. 2018.01.027

Morgan, C., \& Cotten, S. R. (2003). The relationship between Internet activities and depressive symptoms in a sample of college freshmen. CyberPsychology \& Behavior, 6(2), 133-142. doi:10.1089/109493103321640329

Odac1, H., \& Kalkan, M. (2010). Problematic Internet use, loneliness and dating anxiety among young adult university students. Computers \& Education, 55(3), 1091-1097. doi:10.1016/ j.compedu.2010.05.006

Oh, I. S., \& Kim, C. (2009). Meta-analysis on the effects of the prevention and intervention programs for Internet addiction. Journal of Korea Association of Information Education, 13(4), 529-537. doi:10.1002/9781444318852.ch20

Oswald, F. L., \& Plonsky, L. D. (2010). Meta-analysis in second language research: Choices and challenges. Annual Review of Applied Linguistics, 30, 85-110. doi:10.1017/S02671905 10000115

Park, S. M. (2009). A meta-analysis on the effects of adolescent Internet addiction group counseling program in Korea. Korean Journal of Counselling Psychothererapy, 21(3), 607-623. doi:10.15703/kjc.16.3.201506.101

Pies, R. (2009). Should DSM-V designate "Internet addiction" a mental disorder? Psychiatry (Edgmont), 6(2), 31-37. Retrieved from https://www.ncbi.nlm.nih.gov/pmc/articles/PMC2719452/

Rosenberg, M. S. (2005). The file-drawer problem revisited: A general weighted method for calculating fail-safe numbers in meta-analysis. Evolution, 59(2), 464-468. doi:10.1111/j.00143820.2005.tb01004.x

Sawyer, S. M., Azzopardi, P. S., Wickremarathne, D., \& Patton, G. C. (2018). The age of adolescence. The Lancet Child \& 
Adolescent Health, 2(3), 223-228. doi:10.1016/S23524642(18)30022-1

Shapira, N. A., Goldsmith, T. D., Keck, P. E., Jr., Khosla, U. M., \& McElroy, S. L. (2000). Psychiatric features of individuals with problematic Internet use. Journal of Affective Disorders, 57(1-3), 267-272. doi:10.1016/S0165-0327(99) 00107-X

Sharma, M. K., \& Palanichamy, T. S. (2018). Psychosocial interventions for technological addictions. Indian Journal of Psychiatry, 60(Suppl. 4), S541. doi:10.4103/psychiatry. IndianJPsychiatry_40_18

Shin, J. H., \& Jang, M. (2016). Effect of group sandplay therapy to be addicted youth's addiction levels and anxiety. Journal of Symbols \& Sandplay Therapy, 7(1), 39-55. doi:10.12964/ jsst. 160002

Uysal, G., \& Balci, S. (2018). Evaluation of a school-based program for Internet addiction of adolescents in Turkey.
Journal of Addictions Nursing, 29(1), 43-49. doi:10.1097/ JAN.0000000000000211

Vondráčková, P., \& Gabrhelik, R. (2016). Prevention of Internet addiction: A systematic review. Journal of Behavioral Addictions, 5(4), 568-579. doi:10.1556/2006.5.2016.085

Yeun, Y. R., \& Han, S. J. (2016). Effects of psychosocial interventions for school-aged children's Internet addiction, selfcontrol and self-esteem: Meta-analysis. Healthcare Informatics Research, 22(3), 217-230. doi:10.4258/hir.2016.22.3.217

Young, K. S. (1999). Internet addiction: Symptoms, evaluation and treatment. In L. VandeCreek \& T. L. Jackson (Eds.), Innovations in clinical practice: A source book (Vol. 17, pp. 19-31). Sarasota, FL: Professional Resource Press.

Zhang, M. W., Lim, R. B., Lee, C., \& Ho, R. C. (2018). Prevalence of Internet addiction in medical students: A meta-analysis. Academic Psychiatry, 42(1), 88-93. doi:10.1007/s40596-0170794-1 